\title{
Spleen as a Site for Hematopoiesis of a Distinct Antigen Presenting Cell Type
}

\author{
Helen C. O’Neill, Kristin L. Griffiths, Pravin Periasamy, Rebecca A. Hinton, \\ Ying-Ying Hey, Sawang Petvises, and Jonathan K. H. Tan \\ Division of Biomedical Sciences, Research School of Biology, The Australian National University, Canberra, ACT 0200, Australia \\ Correspondence should be addressed to Helen C. O’Neill, helen.oneill@anu.edu.au
}

Received 4 June 2011; Revised 17 August 2011; Accepted 23 August 2011

Academic Editor: Roland Jurecic

Copyright (C) 2011 Helen C. O’Neill et al. This is an open access article distributed under the Creative Commons Attribution License, which permits unrestricted use, distribution, and reproduction in any medium, provided the original work is properly cited.

While spleen and other secondary tissue sites contribute to hematopoiesis, the nature of cells produced and the environment under which this happens are not fully defined. Evidence is reviewed here for hematopoiesis occurring in the spleen microenvironment leading to the production of tissue-specific antigen presenting cells. The novel dendritic-like cell identified in spleen is phenotypically and functionally distinct from other described antigen presenting cells. In order to identify these cells as distinct, it has been necessary to show that their lineage origin and progenitors differ from that of other known dendritic and myeloid cell types. The spleen therefore represents a distinct microenvironment for hematopoiesis of a novel myeloid cell arising from self-renewing hematopoietic stem cells (HSC) or progenitors endogenous to spleen.

\section{Hematopoiesis in the Mouse Model}

Hematopoietic stem cells arise in the embryo from an hemangioblast present in yolk sac and aorta-gonadmesonephros. They then migrate into fetal liver and spleen, entering bone marrow in the late embryonic period [1]. Numbers of HSC increase in bone marrow and spleen in the neonatal period and are then maintained in those organs for life $[2,3]$. HSC migration into bone marrow at birth then determines bone as the dominant site for hematopoiesis in adults. HSC have also been identified in extramedullary sites in the steady state and small numbers also mobilize through blood and lymph into sites like liver, spleen, brain, lung and intestine $[4,5]$. Infection, inflammation, or drug treatment can lead to expansion in the number of hematopoietic stem/progenitor cells (HSPC) circulating in blood and entering tissues like spleen and liver $[6,7]$. Despite the small numbers of HSPC present in extramedullary tissues in the steady state, this fact should not discount their importance or potential contribution to the development of the immune system and immune responsiveness. Findings from this lab indicate that spleen in the steady state contains HSC [2] and that these undergo myelopoiesis within spleen giving rise to a novel type of dendritic-like antigen presenting cell [8].

\section{The Hematopoietic Stem Cell}

Bone marrow is a very rich source of HSC and for decades bone marrow transplants have provided HSC for regeneration of the hematopoietic system in humans. HSC have distinguishing properties of self-renewal, asymmetric cell division, and ability to clonally regenerate all hematopoietic cell types. Methodology for sorting HSC from bone marrow using specific antibodies and flow cytometry [9] has become a standard technique in many labs. HSC are heterogeneous, comprising cells with both long-term and short-term reconstitution capacity $[10,11]$. Long-term reconstituting HSC are commonly isolated from murine bone marrow as lineage (Lin) ${ }^{-} \mathrm{Sca}-1^{+}{ }^{\mathrm{c}}-\mathrm{kit}^{+} \mathrm{Flt} 3^{-}$cells [11] and can be further purified as the $\mathrm{CD} 150^{+} \mathrm{CD} 48^{-}$subset of $\mathrm{Lin}^{-} \mathrm{Scal}^{+} \mathrm{ckit}^{+}$ bone marrow [12, 13]. Epigenetic imprinting of HSC also contributes to differences in hematopoietic potential [14]. Given the level of heterogeneity amongst HSC, it is important to consider them in relation to their tissue niche 
of origin, their distinct hematopoietic potential, and to use this knowledge to inform HSC transplantation in the clinic. Most studies have concentrated on the more prevalent HSC in bone marrow with less attention given to HSC in extramedullary sites like spleen. While HSC from tissue sites like bone marrow, cord blood and spleen can reconstitute all cell lineages, there has been no comprehensive study of their relative capacity to produce a range of hematopoietic cell subsets. The hematopoietic support capacity of different tissue niches is also a factor not yet investigated in terms of hematopoietic output. Recent evidence that spleen supports the development of distinct dendritic-like antigen presenting cells suggests that spleen may contribute in a distinct, tissuespecific manner to hematopoiesis.

\section{The Spleen in Hematopoiesis}

HSC represent a rare subset in adult mouse spleen. They are however readily detectable by their long-term reconstitution ability upon adoptive transfer into irradiated host mice [2, 3] (Figure 1). The weaker contribution of splenic HSC compared with bone-marrow-derived HSC to hematopoiesis in the normal animal has been reported [15]. It has long been assumed that spleen fills the role of an emergency or backup site for cell development at times of stress or disease. Indeed, the importance of bone marrow over spleen in hematopoiesis is evident since neonatally splenectomised mice can maintain normal bone marrow hematopoiesis [16]. However, a distinct role for spleen in hematopoiesis has not yet been fully investigated in terms of development of individual cell subsets. During embryogenesis, spleen harbours myeloid cells seeding from fetal liver and does not appear to become colonized with HSC until around the time of birth [3]. Evidence that splenectomy in newborn mice results in a dramatic increase in colony forming cells in liver suggests that HSC entering spleen at this time may seed directly into spleen from fetal liver rather than from bone marrow [3]. Spleen could therefore become colonized by HSC during development resulting in an endogenous HSC population. Such a model would not however preclude later or additional entry of bone-marrow-derived HSC at times of stress or inflammation, or following stem cell transplantation.

Spleen is also a central organ for development of dendritic cells (DC) that initiate immune responses by taking up and presenting antigen to lymphocytes. Murine spleen contains multiple subsets of DC including the CD $8 \alpha^{-}$ and $\mathrm{CD} 8 \alpha^{+}$conventional(c)DC, plasmacytoid(p)DC [17], and regulatory DC which are not well characterised [18]. Monocyte-derived DC are also found in spleen but only under conditions of inflammation [19]. Very few markers distinguish these subsets and they are best distinguished by their distinct function in immunity. Both $\mathrm{cDC}$ subsets are powerful T-cell activators, although the $\mathrm{CD} 8 \alpha^{-} \mathrm{cDC}$ are located in the marginal zone of spleen where antigen enters from blood, while the $\mathrm{CD} 8 \alpha^{+}$cDC are located in the Tcell areas where they are poised for T-cell stimulation. The $\mathrm{CD} 8 \alpha^{-}$cDC preferentially activate a T-helper(h)2 response with T-cell production of interleukin(IL)-4 and IL-10, while $\mathrm{CD} 8 \alpha^{+}$cDC preferentially induce a Th1 response marked by interferon (IFN)- $\gamma$ production $[20,21]$. While both subsets of $\mathrm{cDC}$ can activate $\mathrm{CD}^{+}$and $\mathrm{CD}^{+} \mathrm{T}$ cells, the $\mathrm{CD} 8 \alpha^{+}$ cDC subset has been shown to be superior at antigen crosspresentation [22]. Plasmacytoid DC are distinct in that they reside mainly in blood in a precursor form, often referred to as p-preDC. They are distinct by their capacity to produce high levels of IFN- $\alpha$ in response to viruses [23]. Regulatory DCs represent a poorly defined class of DCs which are inhibitory rather than stimulatory for $\mathrm{T}$ cells, producing factors like IL-10 and nitric oxide [18, 24]. In contrast to cDC and pDC, mo-DCs are not detectable in spleen in the resting state. They develop from blood monocytes in response to inflammatory factors. Tumour necrosis factor $\alpha$ /inducible nitric oxide synthase producing (Tip) DC derive from monocytes and enter tissues where they function as strong T-cell activators [25].

An essential element of DC biology is the definition of progenitors and precursors since this underpins the formation of lineages of cells with distinct function. Initially $\mathrm{cDC}$ and $\mathrm{pDC}$ progenitors were defined as a Flt $3^{+}$ subset amongst common myeloid and lymphoid progenitors (CMP/CLP) in bone marrow [26]. Recent evidence now points to a monocyte/dendritic progenitor (MDP) in bone marrow which gives rise to all monocyte/macrophage and dendritic type cells $[27,28]$, as well as a more committed common dendritic progenitor (CDP) for $\mathrm{CDC}$ and $\mathrm{pDC}$ in bone marrow $[29,30]$. While CDP and MDP are not present in spleen or blood [31], spleen does harbour more differentiated $\mathrm{CDC}$ precursors which have a high turnover and are replaced by blood-borne precursors migrating from bone marrow [32]. Studies with parabiotic mice that share a circulatory system, suggest that some splenic DC might arise from endogenous progenitors [33], although others have questioned that result [34]. The presence of an endogenous hematopoietic progenitor in spleen that differentiates to give a novel and distinct dendritic-like cell is now indicated by the findings described below.

\section{A Novel Antigen Presenting Cell Subset in Spleen}

Distinct myeloid dendritic-like cells have been discovered as a subset in murine spleen [35] on the basis of similarity with dendritic-like cells identified previously which develop in splenic longterm cultures (LTC) [36-38]. LTC-derived DC and the in vivo subset of "L-DC", have a characteristic immature phenotype as $\mathrm{CD} 11 \mathrm{c}^{\mathrm{lo}} \mathrm{CD} 11 \mathrm{~b}^{\text {hi }} \mathrm{MHC}-\mathrm{II}^{-} \mathrm{CD} 8 \alpha^{-}$cells, distinguishing them from $\mathrm{cDC}, \mathrm{pDC}$, and monocytes [35] (Figure 2). They are large, endocytic cells specialized in crosspresentation of antigen to $\mathrm{CD}^{+} \mathrm{T}$ cells, a function usually attributed to $\mathrm{CD} 8 \alpha^{+}$cDC [39]. LTC-DC are also distinct by their very weak ability to activate $\mathrm{CD}^{+}{ }^{+} \mathrm{T}$ cells and their $\mathrm{MHC}^{-} \mathrm{II}^{-}$phenotype, properties which distinguish them from all of the common subsets of CD $8 \alpha^{+} \mathrm{cDC}, \mathrm{CD} 8 \alpha^{-} \mathrm{cDC}$, and $\mathrm{pDC}[35,37,40]$. Since LTC-DC can be derived from $G M-C S F^{-/-}$mice [41], they do not resemble monocytederived DC that develop in response to inflammatory factors like GM-CSF and TNF- $\alpha$ [19]. 


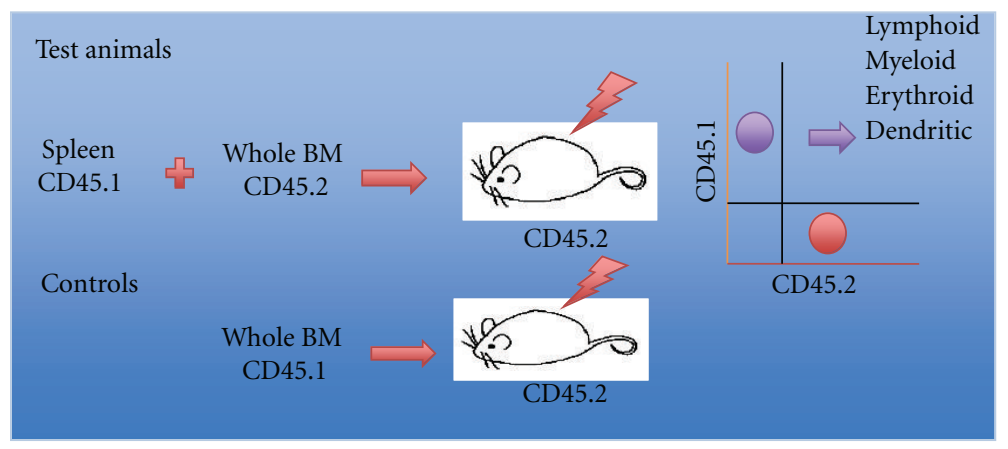

FIGURE 1: Hematopoietic reconstitution assay for detecting the presence of HSC. The hematopoietic system of lethally irradiated host mice can be fully reconstituted with lymphoid, myeloid, erythroid, and dendritic cells following adoptive transfer of bone marrow cells as a source of HSC. In order to assess the presence of HSC or other hematopoietic progenitors in a test population of cells, CD45-allotype distinct cells are adoptively transferred with assessment of the hematopoietic reconstitution capacity of this specific population by flow cytometry. In most cases, test animals are also given an inoculum of host type bone marrow to ensure survival. HSC with long-term reconstitution potential produce progeny out to 25 weeks, while HSC with short-term potential are assessed after 8 weeks.

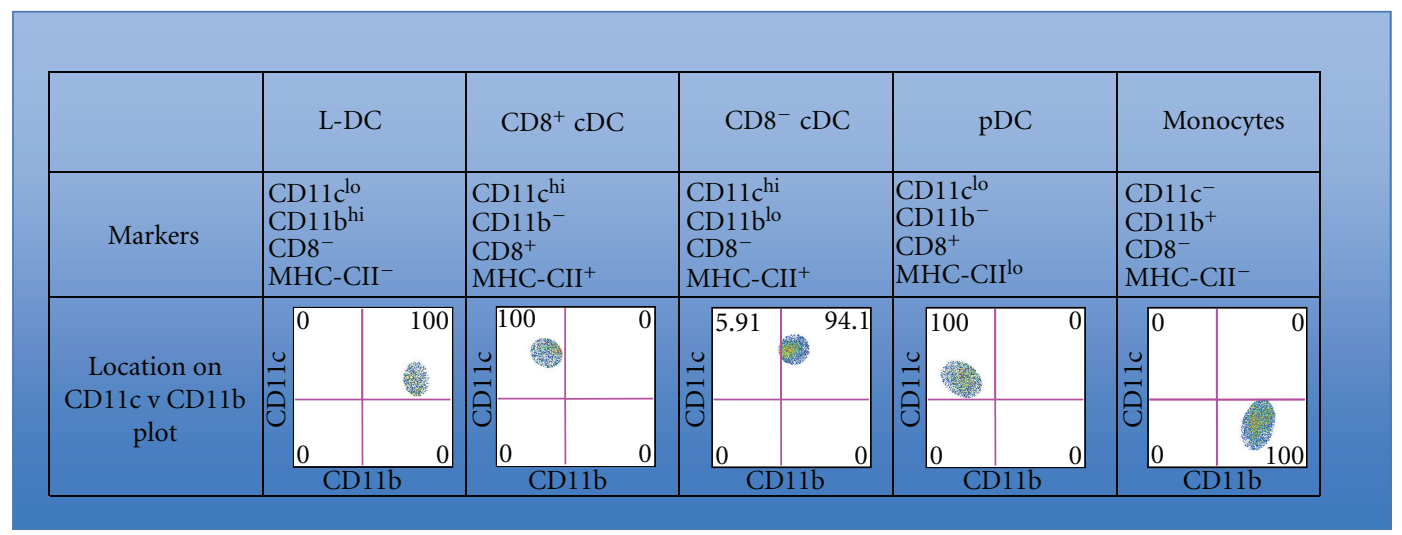

FIGURE 2: Classification of DC and myeloid subsets in spleen. Subsets in spleen can be identified flow cytometrically in terms of their phenotype following staining with specific antibody to cell surface markers. The different subsets can be identified based on variant expression of CD11c, CD11b, CD8 $\alpha$ and MHC-II. The phenotype of L-DC is shown by comparison with pDC, cDC and a broad monocyte population. The different subsets can also be located in distinct positions on a bivariate CD11c versus CD11b scatter plot.

An isolation procedure was optimised to reveal the in vivo equivalent cell type to LTC-DC [35]. This procedure distinguished $\mathrm{CD} 8 \alpha^{+} \mathrm{cDC}, \mathrm{CD} 8 \alpha^{-} \mathrm{cDC}, \mathrm{pDC}$ and monocytes on the basis of CD11b, CD11c, MHC-II and $\mathrm{CD} 8 \alpha$ expression, from a population of cells of large size (FSC ${ }^{\text {hi }}$ ) resembling LTC-DC by their CD $11 \mathrm{c}^{\mathrm{lo}} \mathrm{CD} 11 \mathrm{~b}^{\text {hi }} \mathrm{MHC}$ $\mathrm{II}^{-} \mathrm{CD} 8 \alpha^{-}$phenotype and their high endocytic capacity for soluble antigen (Figure 3 ). These cells have been tentatively named "L-DC", since they resemble a DC more than a myeloid cell. L-DC are phenotypically and functionally distinct from monocytes/macrophages, $\mathrm{CDC}$ and $\mathrm{pDC}$. They are distinct as $\mathrm{CD} 11 \mathrm{c}^{+}$cells that are highly cross-presenting, a property which distinguishes them from common subsets of macrophages and monocytes [40]. Recent evidence for uniform expression of F4/80 and CD11c, but not Ly6G, also distinguishes L-DC from neutrophils [42]. The immune potential of this in vivo subset is under continued investigation in terms of both T-cell and B-cell immunity. Already we know that L-DC share similar antigen cross-presenting function for $\mathrm{CD}^{+}$T-cell activation with LTC-DC [35]. Like
LTC-DC, they are also unable to activate $\mathrm{CD}^{+}{ }^{+} \mathrm{T}$ cells, consistent with the absence of MHC-II expression [37, 40]. Despite their distinct functional potential, the lineage origin of L-DC relative to other known splenic myeloid and DC subsets, is still unknown.

The distinctiveness of L-DC from monocytes, cDC subsets and granulocytes has been confirmed by transcriptome analysis of subsets sorted as shown in Figure 3. Principal Components Analysis of the data indicated a broad separation of overall gene expression between LDC and other subsets, with $\mathrm{CD} 8 \alpha^{+}$and $\mathrm{CD} 8 \alpha^{-} \mathrm{CDC}$ the most closely related subsets (Figure 4). L-DC specifically express high levels of Treml4, a gene recently identified as expressed by $\mathrm{CD} 8 \alpha^{+}$but not $\mathrm{CD} 8 \alpha^{-} \mathrm{CDC}$ and by splenic red pulp and metallophilic macrophages [43]. This gene encodes a receptor that binds late apoptotic and necrotic cells, consistent with cross-presenting capacity related to the uptake of dead and dying cells from blood. The relationship between L-DC and other DC and macrophage cell types is currently under investigation. 


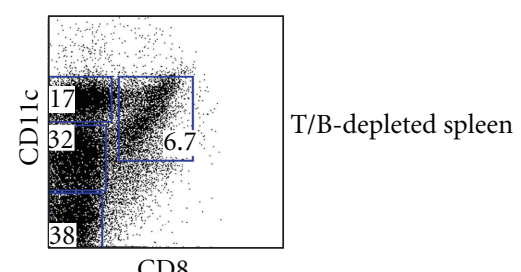

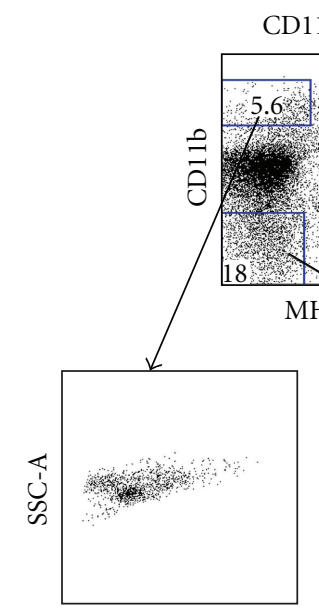

FSC-A

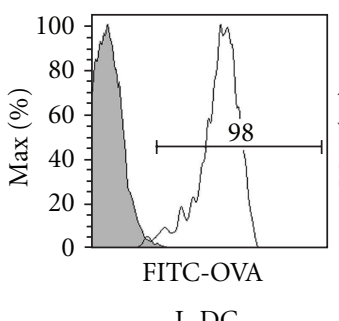

L-DC

CD8

D11 ${ }^{\text {lo }} \mathrm{CD} 8^{-}$

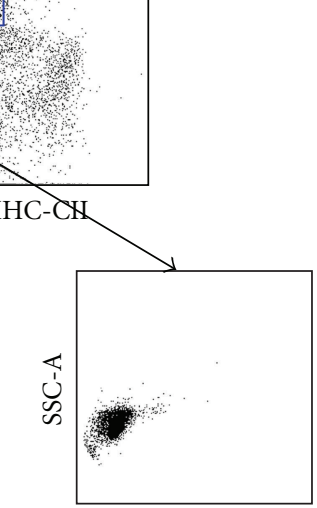

FSC-A

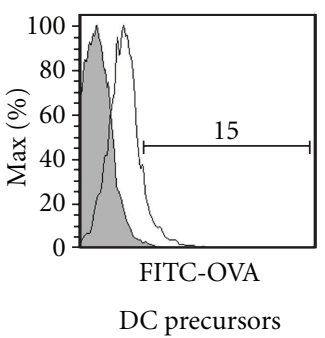

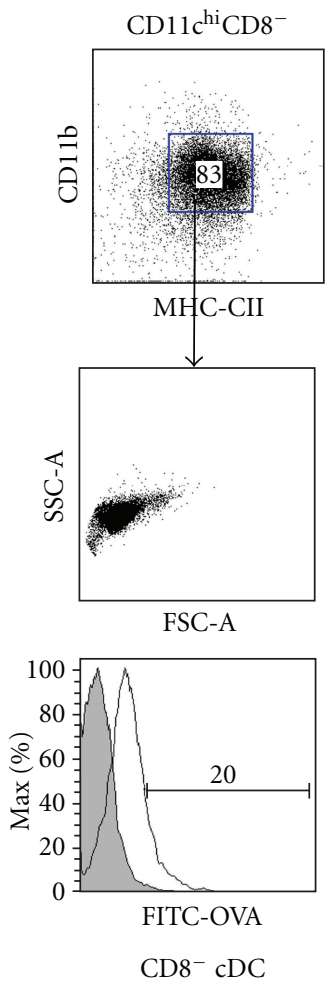

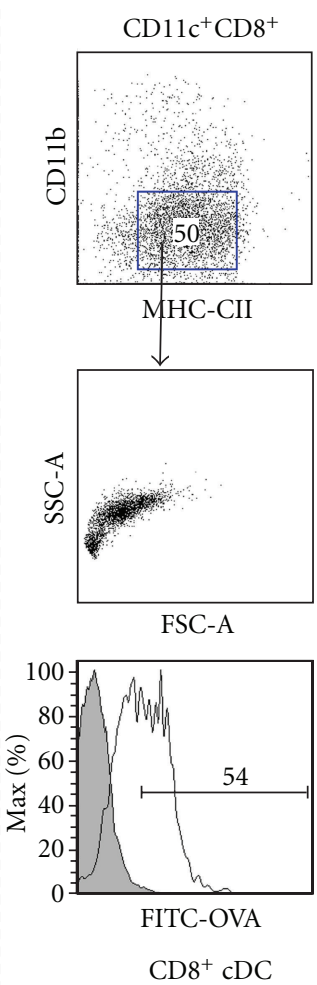

Figure 3: Isolation of L-DC (LIVE-DC) in spleen amongst other DC subsets. Spleens were depleted of T and B cells using antibody-coated magnetic beads and then stained with antibodies specific for CD11c, CD11b, CD8 $\alpha$ and MHC-II ahead of flow cytometric analysis. DC subsets were identified based on multiple parameters of forward scatter (FSC), side scatter (SSC), marker expression and endocytosis after intravenous administration of FITC-OVA $(0.6 \mathrm{mg} / \mathrm{mouse}) 24$ hours previously.

\section{Hematopoiesis Leading to L-DC Production In Vitro}

Coculture assays are now used instead of LTC to produce $\mathrm{L}-\mathrm{DC}$ in vitro. These involve overlay of $\mathrm{Lin}^{-} \mathrm{BM}$ or T/Bcell-depleted spleen cell suspensions over competent splenic stroma [40]. The splenic stromal line STX3 and its 5G3 clone originated from a long-term splenic culture that had lost hematopoietic progenitors over time and passage [40]. The 5G3 clone of STX3 has been characterized as an immature endothelial-like cell $[44,45]$, unique in its phenotype and function as a supporter of in vitro hematopoiesis of L-DC. There also appears to be no equivalent stromal cell line reported in the literature. Cells produced in cocultures over 35 days both phenotypically and functionally resemble LTC$\mathrm{DC}$ as well as the in vivo L-DC subset $[8,40]$. Cocultures can therefore be used to identify L-DC progenitors amongst sorted cell subsets by their capacity to seed stroma for continued L-DC production in vitro. Indeed, the continuous nature of LTC which produce dendritic-like cells over several years [36], as well as the ability of STX3 or 5G3 stroma to support in vitro hematopoiesis of L-DC for up to a year [46], suggests that the L-DC progenitor may represent a selfrenewing HSPC.

The development of L-DC in LTC depends on the maintenance of a population of small progenitors [38, 47]. In fact, the requirement for differentiation of progenitors was demonstrated by sorting the small progenitor population in LTC and showing that these cells produced L-DC upon transfer to stromal cocultures [38]. L-DC were however shown to undergo several rounds of proliferation in bromodeoxyuridine labeling experiments [47]. The production of dendritic-like cells by differentiation from HSPC maintained within LTC as opposed to proliferation of L-DC was also confirmed by gene profiling of subtracted libraries prepared between the purified small and large cells maintained in LTC [48]. These studies showed that small cells resemble HSPC, while large cells reflect myeloid dendritic cells. More 


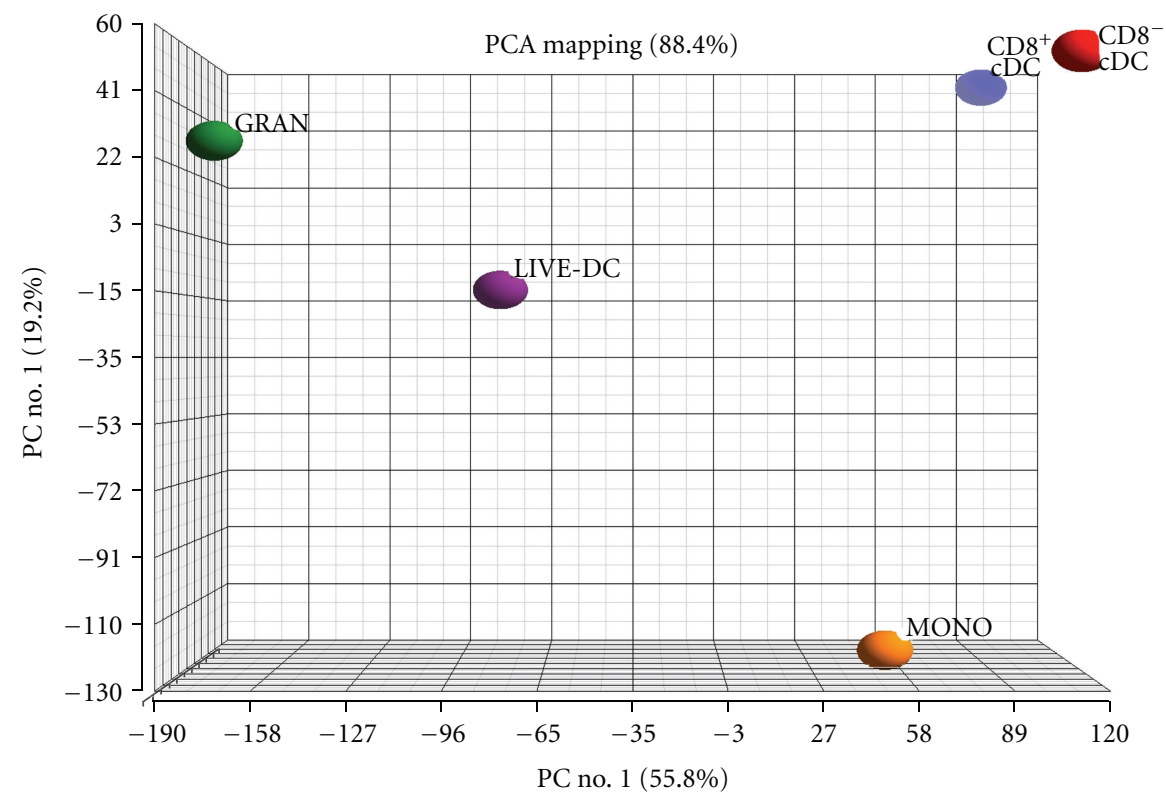

FIGURE 4: Relatedness of transcriptome between L-DC (LIVE-DC) and other spleen myeloid subsets. Myeloid and DC subsets were isolated from spleen by antibody staining and cell sorting using flow cytometry as demonstrated in Figure 3. RNA was prepared from DC subsets described in Figure 2, and from granulocytes (GRAN) as Gr- $1^{+} \mathrm{CD} 11 \mathrm{~b}^{+} \mathrm{CD} 11 \mathrm{c}^{-}$cells and monocytes (MONO) as CD11 $\mathrm{b}^{+} \mathrm{CD} 11 \mathrm{c}^{-} \mathrm{MHC}^{-\mathrm{II}^{-}}$ cells. RNA was used to probe Affymetrix Gene 1.0ST microarrays. Data show Principal Components Analysis (1\&2) reflecting degree of relatedness in overall gene expression between subsets.

recent studies have involved phenotypic characterization of progenitors. Over many experiments, progenitor activity was found to be enriched within the subset of $\mathrm{Lin}^{-} \mathrm{ckit}^{+} \mathrm{CD} 34^{-/+}$ "small" cells in LTC, a phenotype reflective of described subsets of HSC differing in terms of long-term or shortterm reconstituting capacity [11]. Highly purified, sorted HSC from bone marrow have now been shown to seed STX3 cocultures to produce L-DC, but only when contact is maintained with stroma [46]. Cells produced were found to phenotypically resemble L-DC. Overall, these results align the L-DC progenitor with the HSC subset in bone marrow. This finding is particularly important in terms of the relationship between the L-DC progenitor and the progenitors of $\mathrm{CDC}$ and $\mathrm{pDC}$ which have been identified as MDP, CDP and myeloid progenitors (MP), which are present in bone marrow but not spleen [31]. One hypothesis is that splenic niches are distinct from bone marrow niches and can support the direct differentiation of HSC to give L-DC in the apparent absence of committed myeloid or dendritic progenitors like the MDP, CDP and MP. The relationship between these progenitors and a putative LDC progenitor in spleen is not known and warrants further investigation.

\section{Identification of L-DC Progenitors in Spleen}

To delineate the L-DC progenitor in mouse spleen, Lin $^{-}$ splenocytes were assessed for expression of ckit and other markers of early hematopoietic progenitors followed by sorting of subsets of interest. Sorted subsets were each assessed for their hematopoietic potential in cocultures of competent stroma. They were also tested in vivo for capacity to reconstitute lethally irradiated CD45-allotype distinct host mice also given syngeneic bone marrow to aid recovery [8] (Figure 1). The Lin ${ }^{-}$ckit $^{\text {lo }}$ spleen subset produced exclusively L-DC in cocultures, and the $\mathrm{Lin}^{-}$ckithi subset produced predominantly L-DC with a few cDC-like cells that were not maintained over time. However, only Lin $^{-}$ckit ${ }^{\text {hi }}$ splenocytes, and not the $\mathrm{Lin}^{-} \mathrm{ckit}^{\mathrm{lo}}$ or $\mathrm{Lin}^{-} \mathrm{ckit}^{-}$subsets, showed multilineage reconstitution in vivo consistent with the presence of HSC [8]. The $\mathrm{Lin}^{-}$ckit ${ }^{\text {hi }}$ population of spleen therefore contains long-term reconstituting $\mathrm{HSC}$ as well as L-DC progenitors, while the $\mathrm{Lin}^{-}$ckit $^{\text {lo }}$ fraction contains only LDC progenitors. One explanation is that there is a common progenitor within each of these two populations. Another is that there are distinct progenitors within the two subsets and that the stroma supports differentiation of ckit ${ }^{\text {hi }}$ progenitors to give $\mathrm{ckit}^{\mathrm{lo}}$ progenitors. The relationship between the LDC progenitor and the HSC subset in spleen is unclear at present. These findings are also consistent with evidence that bone-marrow-derived HSC can seed stroma for L-DC production and that the L-DC progenitor maintained in LTC is represented by a $\mathrm{Lin}^{-} \mathrm{ckit}^{+} \mathrm{CD} 34^{+/-}$subset. Splenic stroma therefore appears to impose specific differentiative capacity on HSC, irrespective of their origin from spleen or bone marrow. In terms of the characterization of LDC progenitors endogenous to spleen, a first goal will be to fractionate subsets of splenic $\mathrm{Lin}^{-} \mathrm{ckit}^{+}$cells further and to identify the minimum subset that produces L-DC in cocultures. 


\section{Does Hematopoiesis in Spleen Involve an Endogenous Progenitor?}

Many studies now indicate a specific role for splenic niches in the development of the novel antigen presenting cell subset, namely L-DC. While the in vitro evidence is direct and compelling, it is a harder task to delineate L-DC production in vivo as dependent on hematopoiesis occurring in spleen and arising from an HSPC endogenous to spleen. In experiments involving adoptive transfer of stem cells into irradiated mice (Figure 1), the issue of where in the animal hematopoiesis occurs is not clear. Transferred stem cells can migrate to and localize in multiple niches in spleen and bone marrow. In these sites, they can undergo hematopoiesis and then migrate into other lymphoid areas. Another more direct method to delineate hematopoiesis specific to splenic HSC involves ectopic grafting of allotype-distinct spleen or spleen fragments under the kidney capsule of splenectomized mice and monitoring the output of donor graft-derived mature cells on the basis of allotype markers. We recently published successful engraftment of neonatal spleen fragments under the kidney capsule of splenectomized mice [49]. Most grafts retained donor-type HSC and showed development of some donor-type myeloid cells at 2 weeks. However, recruitment of host HSC, lymphocytes and myeloid cells into grafts was also evident [49]. The spleen microenvironment therefore appears to support myelopoiesis and possibly LDC development. The development of individual subsets of myeloid cells is yet to be analysed more completely using this model.

Indirect evidence that spleen supports hematopoiesis and the specific differentiation of L-DC also comes from chimera studies. These studies compared the relative ability of splenic versus bone marrow HSC to generate the different myeloid and DC subsets including L-DC [2]. In several studies, analysis of the relative numbers of donor:host progeny cells representing each of the common DC and myeloid subsets produced in chimeras identified a distinct developmental bias towards donor L-DC over other myeloid and DC subsets [2]. The only explanation for this finding is that splenic niches preferentially support the development of L-DC from HSC. Another consideration is whether the microenvironment in which HSC develop conditions their hematopoietic capacity. In terms of stem cell transplantation, it will be important to determine whether HSC arising in $\mathrm{BM}$, spleen, or fetal liver can adopt distinct differentiative capacity after residence in splenic niches.

\section{Conclusion}

A history of published work now supports the hypothesis that the splenic L-DC subset described here represents a functionally distinct tissue-specific antigen presenting cell type that develops within spleen from endogenous HSPC. Its development is entirely dependent on endothelial niches within spleen although bone-marrow-derived HSC can also differentiate within this niche to produce L-DC. The hypothesis that spleen contains an endogenous HSC laid down during embryogenesis would be consistent with evidence for tissue-specific hematopoiesis of L-DC.

The identification of a novel antigen presenting cell subset with unique immune function is of significant immunological interest. It is therefore important to define the hematopoietic pathways in spleen that lead to development of these dendritic-like cells, especially since these cells do not appear to have a lineage origin in common with other described cDC and myeloid subsets. Indeed, there are now a number of examples in the literature of tissuespecific antigen presenting cells developing from endogenous progenitors maintained in distinct tissue sites. For example, Langerhans cells in skin arise from epidermal progenitors that colonize embryonic skin [50], and microglia represent antigen presenting cells in brain which develop from selfrenewing progenitors induced to proliferate after inflammation [51]. These examples support a model for compartmentalization of the immune response by the production of tissue-specific antigen presenting cells, and specialization of immunity in tissues colonized by particular pathogens. A full understanding of hematopoiesis occurring in extramedullary sites will be essential knowledge for therapeutic practice in immunotherapy and indeed in HSC transplantation.
Abbreviations
HSC: Hematopoietic stem cells
HSPC: Hematopoietic stem/progenitor cells
DC: Dendritic cells
cDC: Conventional DC
pDC: Plasmacytoid DC
CMP: Common myeloid progenitors
CLP: Common lymphoid progenitors
CDP: Common dendritic progenitors
MDP: Myeloid/dendritic progenitors
MP: $\quad$ Myeloid progenitors.

\section{Conflict of Interests}

The authors declare no financial or commercial conflict of interests.

\section{Acknowledgment}

This work was supported in part by funding from the National Health and Medical Research Council of Australia (Project Grant no. 585443).

\section{References}

[1] J. L. Christensen, D. E. Wright, A. J. Wagers, and I. L. Weissman, "Circulation and chemotaxis of fetal hematopoietic stem cells," PLoS Biology, vol. 2, no. 3, p. E75, 2004.

[2] J. K. Tan and H. C. O’Neill, “Haematopoietic stem cells in spleen have distinct differentiative potential for antigen presenting cells," Journal of Cellular and Molecular Medicine, vol. 14, no. 8, pp. 2144-2150, 2010.

[3] F. M. Wolber, E. Leonard, S. Michael, C. M. OrschellTraycoff, M. C. Yoder, and E. F. Srour, "Roles of spleen and 
liver in development of the murine hematopoietic system," Experimental Hematology, vol. 30, no. 9, pp. 1010-1019, 2002.

[4] S. Massberg, P. Schaerli, I. Knezevic-Maramica et al., "Immunosurveillance by hematopoietic progenitor cells trafficking through blood, lymph, and peripheral tissues," Cell, vol. 131, no. 5, pp. 994-1008, 2007.

[5] D. E. Wright, S. H. Cheshier, A. J. Wagers, T. D. Randall, J. L. Christensen, and I. L. Weissman, "Cyclophosphamide/granulocyte colony-stimulating factor causes selective mobilization of bone marrow hematopoietic stem cells into the blood after M phase of the cell cycle," Blood, vol. 97, no. 8, pp. 2278-2285, 2001.

[6] R. A. Abrams, A. Johnston-Early, C. Kramer, J. D. Minna, M. H. Cohen, and A. B. Deisseroth, "Amplification of circulating granulocyte-monocyte stem cell numbers following chemotherapy in patients with extensive small cell carcinoma of the lung," Cancer Research, vol. 41, no. 1, pp. 35-41, 1981.

[7] C. M. Richman, R. S. Weiner, and R. A. Yankee, "Increase in circulating stem cells following chemotherapy in man," Blood, vol. 47, no. 6, pp. 1031-1039, 1976.

[8] J. K. Tan, P. Periasamy, and H. C. O'Neill, "Delineation of precursors in murine spleen that develop in contact with splenic endothelium to give novel dendritic-like cells," Blood, vol. 115, no. 18, pp. 3678-3685, 2010.

[9] G. J. Spangrude, S. Heimfeld, and I. L. Weissman, "Purification and characterization of mouse hematopoietic stem cells," Science, vol. 241, no. 4861, pp. 58-62, 1988.

[10] A. Foudi, K. Hochedlinger, D. van Buren et al., "Analysis of histone 2B-GFP retention reveals slowly cycling hematopoietic stem cells," Nature Biotechnology, vol. 27, no. 1, pp. 84-90, 2009.

[11] A. Y. Lai, S. M. Lin, and M. Kondo, "Heterogeneity of Flt3expressing multipotent progenitors in mouse bone marrow," Journal of Immunology, vol. 175, no. 8, pp. 5016-5023, 2005.

[12] M. J. Kiel, Ö. H. Yilmaz, T. Iwashita, O. H. Yilmaz, C. Terhorst, and S. J. Morrison, "SLAM family receptors distinguish hematopoietic stem and progenitor cells and reveal endothelial niches for stem cells," Cell, vol. 121, no. 7, pp. 1109-1121, 2005.

[13] P. Papathanasiou, J. L. Attema, H. Karsunky, X. Jian, S. T. Smale, and I. L. Weissman, "Evaluation of the longterm reconstituting subset of hematopoietic stem cells with CD150," Stem Cells, vol. 27, no. 10, pp. 2498-2508, 2009.

[14] J. L. Attema, P. Papathanasiou, E. C. Forsberg, J. Xu, S. T. Smale, and I. L. Weissman, "Epigenetic characterization of hematopoietic stem cell differentiation using miniChIP and bisulfite sequencing analysis," Proceedings of the National Academy of Sciences of the United States of America, vol. 104, no. 30, pp. 12371-12376, 2007.

[15] Y. Morita, A. Iseki, S. Okamura, S. Suzuki, H. Nakauchi, and H. Ema, "Functional characterization of hematopoietic stem cells in the spleen," Experimental Hematology, vol. 39, no. 3, pp. 351-359.e3, 2011.

[16] A. M. Shatry and R. B. Levy, "Engraftment of splenic tissue as a method to investigate repopulation by hematopoietic cells from host and donor marrow," Stem Cells and Development, vol. 13, no. 4, pp. 390-399, 2004.

[17] K. Shortman and S. H. Naik, "Steady-state and inflammatory dendritic-cell development," Nature Reviews Immunology, vol. 7, no. 1, pp. 19-30, 2007.

[18] M. Svensson, A. Maroof, M. Ato, and P. M. Kaye, "Stromal cells direct local differentiation of regulatory dendritic cells," Immunity, vol. 21, no. 6, pp. 805-816, 2004.
[19] C. Cheong, I. Matos, J. H. Choi et al., "Microbial stimulation fully differentiates monocytes to DC-SIGN/CD209+ dendritic cells for immune T cell areas," Cell, vol. 143, no. 3, pp. 416429, 2010.

[20] R. Maldonado-López, T. de Smedt, P. Michel et al., "CD8 $\alpha^{+}$ and CD $8 \alpha^{-}$subclasses of dendritic cells direct the development of distinct T helper cells in vivo," Journal of Experimental Medicine, vol. 189, no. 3, pp. 587-592, 1999.

[21] B. Pulendran, J. L. Smith, G. Caspary et al., "Distinct dendritic cell subsets differentially regulate the class of immune response in vivo," Proceedings of the National Academy of Sciences of the United States of America, vol. 96, no. 3, pp. 1036-1041, 1999.

[22] J. L. Pooley, W. R. Heath, and K. Shortman, "Cutting edge: intravenous soluble antigen is presented to CD4 $\mathrm{T}$ cells by $\mathrm{CD}^{-}$dendritic cells, but cross-presented to CD8 $\mathrm{T}$ cells by CD8 ${ }^{+}$dendritic cells," Journal of Immunology, vol. 166, no. 9, pp. 5327-5330, 2001.

[23] C. Asselin-Paturel, A. Boonstra, M. Dalod et al., "Mouse type I IFN-producing cells are immature APCs with plasmacytoid morphology," Nature Immunology, vol. 2, no. 12, pp. 11441150, 2001.

[24] M. Zhang, H. Tang, Z. Guo et al., "Splenic stroma drives mature dendritic cells to differentiate into regulatory dendritic cells," Nature Immunology, vol. 5, no. 11, pp. 1124-1133, 2004.

[25] N. V. Serbina, T. P. Salazar-Mather, C. A. Biron, W. A. Kuziel, and E. G. Pamer, "TNF/iNOS-producing dendritic cells mediate innate immune defense against bacterial infection," Immunity, vol. 19, no. 1, pp. 59-70, 2003.

[26] A. d'Amico and L. Wu, "The early progenitors of mouse dendritic cells and plasmacytoid predendritic cells are within the bone marrow hemopoietic precursors expressing Flt3," Journal of Experimental Medicine, vol. 198, no. 2, pp. 293-303, 2003.

[27] D. K. Fogg, C. Sibon, C. Miled et al., "A clonogenic bone marrow progenitor specific for macrophages and dendritic cells," Science, vol. 311, no. 5757, pp. 83-87, 2006.

[28] F. Geissmann, M. G. Manz, S. Jung, M. H. Sieweke, M. Merad, and K. Ley, "Development of monocytes, macrophages, and dendritic cells," Science, vol. 327, no. 5966, pp. 656-661, 2010.

[29] S. H. Naik, P. Sathe, H. Y. Park et al., "Development of plasmacytoid and conventional dendritic cell subtypes from single precursor cells derived in vitro and in vivo," Nature Immunology, vol. 8, no. 11, pp. 1217-1226, 2007.

[30] N. Onai, A. Obata-Onai, M. A. Schmid, T. Ohteki, D. Jarrossay, and M. G. Manz, "Identification of clonogenic common Flt3+M-CSFR+ plasmacytoid and conventional dendritic cell progenitors in mouse bone marrow," Nature Immunology, vol. 8, no. 11, pp. 1207-1216, 2007.

[31] K. Liu, G. D. Victora, T. A. Schwickert et al., "In vivo analysis of dendritic cell development and homeostasis," Science, vol. 324, no. 5925, pp. 392-397, 2009.

[32] S. H. Naik, D. Metcalf, A. van Nieuwenhuijze et al., "Intrasplenic steady-state dendritic cell precursors that are distinct from monocytes," Nature Immunology, vol. 7, no. 6, pp. 663-671, 2006.

[33] K. Kabashima, T. A. Banks, K. M. Ansel, T. T. Lu, C. F. Ware, and J. G. Cyster, "Intrinsic lymphotoxin- $\beta$ receptor requirement for homeostasis of lymphoid tissue dendritic cells," Immunity, vol. 22, no. 4, pp. 439-450, 2005.

[34] K. Liu, C. Waskow, X. Liu, K. Yao, J. Hoh, and M. Nussenzweig, "Origin of dendritic cells in peripheral lymphoid organs of mice,” Nature Immunology, vol. 8, no. 6, pp. 578-583, 2007. 
[35] J. K. Tan, B. J. Quah, K. L. Griffiths, P. Periasamy, Y. Y. Hey, and H. C. O'Neill, "Identification of a novel antigen cross-presenting cell type in spleen," Journal of Cellular and Molecular Medicine, vol. 15, no. 5, pp. 1189-1199, 2011.

[36] H. C. O’Neill, H. L. Wilson, B. Quah, J. L. Abbey, G. Despars, and $\mathrm{K} . \mathrm{Ni}$, "Dendritic cell development in long-term spleen stromal cultures," Stem Cells, vol. 22, no. 4, pp. 475-486, 2004.

[37] B. Quah, K. Ni, and H. C. O’Neill, “In vitro hematopoiesis produces a distinct class of immature dendritic cells from spleen progenitors with limeted T cell stimulation," International Immunology, vol. 16, no. 4, pp. 567-577, 2004.

[38] H. L. Wilson, K. Ni, and H. C. O'Neill, "Identification of progenitor cells in long-term spleen stromal cultures that produce immature dendritic cells," Proceedings of the National Academy of Sciences of the United States of America, vol. 97, no. 9, pp. 4784-4789, 2000.

[39] G. T. Belz, C. M. Smith, L. Kleinert et al., "Distinct migrating and nonmigrating dendritic cell population are involved in MHC class I-restricted antigen presentation after lung infection with virus," Proceedings of the National Academy of Sciences of the United States of America, vol. 101, no. 23, pp. 8670-8675, 2004.

[40] P. Periasamy, J. K. Tan, K. L. Griffiths, and H. C. O’Neill, "Splenic stromal niches support hematopoiesis of dendriticlike cells from precursors in bone marrow and spleen," Experimental Hematology, vol. 37, no. 9, pp. 1060-1071, 2009.

[41] K. Ni and H. C. O’Neill, "Development of dendritic cells from GM-CSF-/- mice in vitro: GM-CSF enhances production and survival of cells," Developmental Immunology, vol. 8, no. 2, pp. 133-146, 2001.

[42] C. Beauvillain, Y. Delneste, M. Scotet et al., "Neutrophils efficiently cross-prime naive T cells in vivo," Blood, vol. 110, no. 8, pp. 2965-2973, 2007.

[43] H. Hemmi, J. Idoyaga, K. Suda et al., "A new triggering receptor expressed on myeloid cells (Trem) family member, trem-like 4 , binds to dead cells and is a DNAX activation protein 12-linked marker for subsets of mouse macrophages and dendritic cells," Journal of Immunology, vol. 182, no. 3, pp. 1278-1286, 2009.

[44] G. Despars, K. Ni, A. Bouchard, T. J. O’Neill, and H. C. O’Neill, "Molecular definition of an in vitro niche for dendritic cell development," Experimental Hematology, vol. 32, no. 12, pp. 1182-1193, 2004.

[45] G. Despars and H. C. O'Neill, "Splenic endothelial cell lines support development of dendritic cells from bone marrow," Stem Cells, vol. 24, no. 6, pp. 1496-1504, 2006.

[46] R. A. Himton and H. C. O’Neill, “In vitro production of distinct deudvitic-like antigen presenting cells from selfrenewing hematopoietre stem cells," Journal of Leukocyte Biology. In press.

[47] H. L. Wilson and H. C. O’Neill, “Dynamics of dendritic cell development from precursors maintained in stromadependent long-term cultures," Immunology and Cell Biology, vol. 81, no. 2, pp. 144-151, 2003.

[48] H. L. Wilson and H. C. O’Neill, "Identification of differentially expressed genes representing dendritic cell precursors and their progeny," Blood, vol. 102, no. 5, pp. 1661-1669, 2003.

[49] J. K. H. Tan and H. C. O'Neill, "Investigation of murine spleen as a niche for hematopoiesis," Transplantation, vol. 89, no. 2, pp. 140-145, 2010.

[50] M. Merad, M. G. Manz, H. Karsunky et al., "Langerhans cells renew in the skin throughout life under steady-state conditions," Nature Immunology, vol. 3, no. 12, pp. 1135-1141, 2002 .
[51] B. Ajami, J. L. Bennett, C. Krieger, W. Tetzlaff, and F. M. Rossi, "Local self-renewal can sustain CNS microglia maintenance and function throughout adult life," Nature Neuroscience, vol. 10, no. 12, pp. 1538-1543, 2007. 

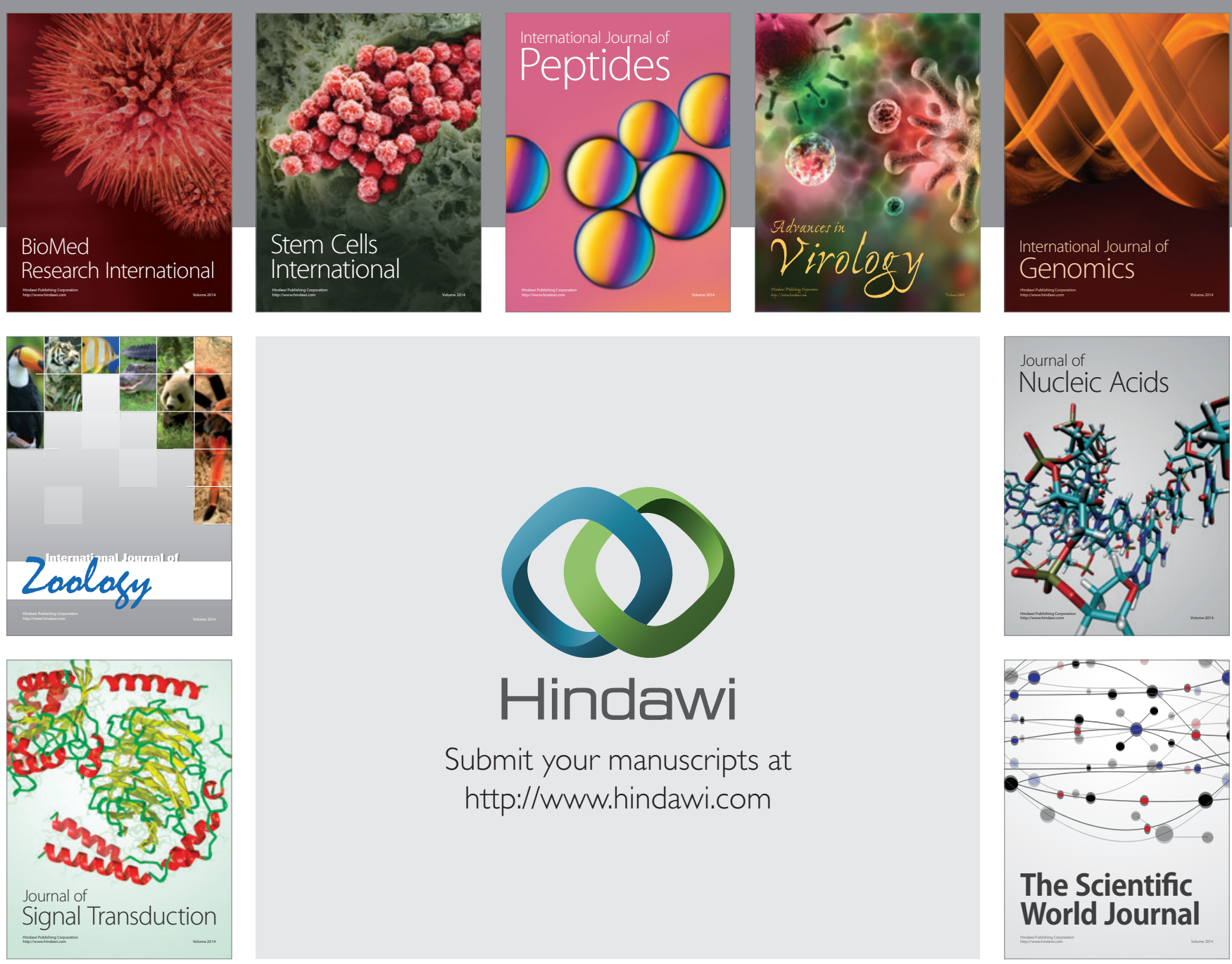

Submit your manuscripts at

http://www.hindawi.com
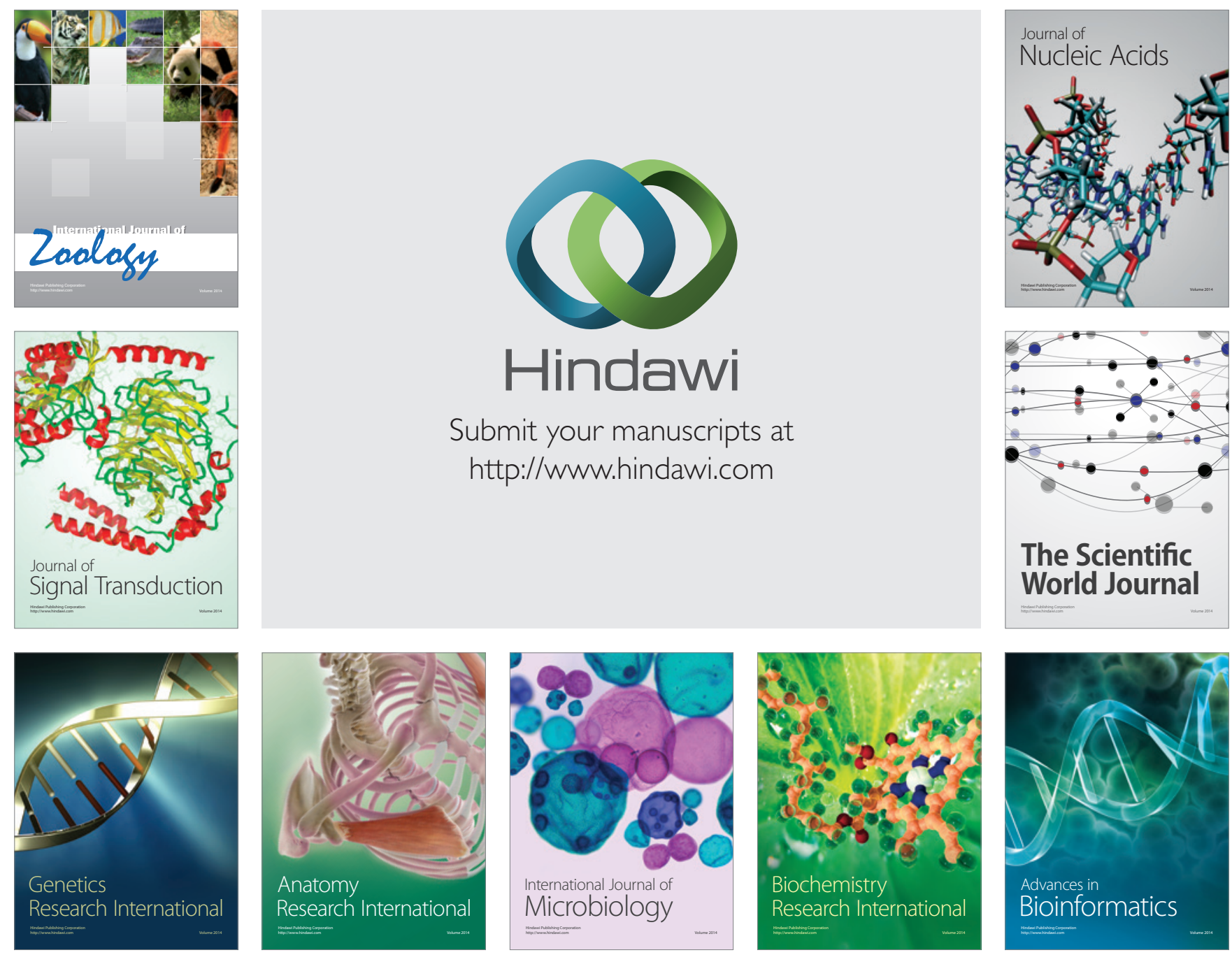

The Scientific World Journal
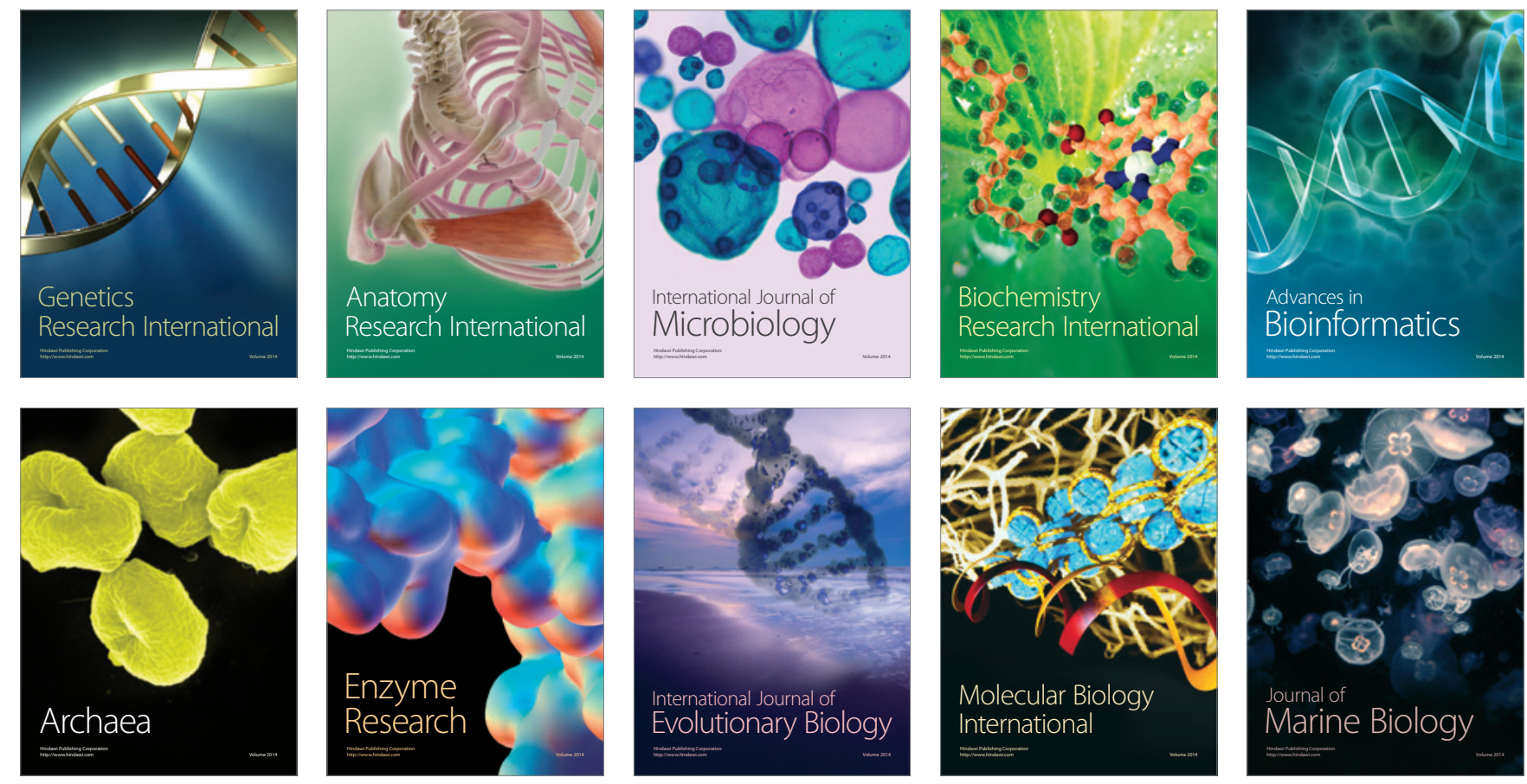